\title{
Uniformity Model for Energetic Ion Processes Using a Kaufman Ion Source
}

\author{
Douglas J. Smith, Joy A. Warner, Nelson LeBarron, \\ Laboratory for Laser Energetics - University of Rochester \\ 250 East River Road \\ Rochester, NY 14623 \\ Phone (716) 275-5101; Fax: (716) 273-1014 \\ dsmi@1le.rochester.edu
}

Processes that use energetic ions for large substrates require that the time-averaged erosion effects from the ion flux be uniform across the surface. A numerical model has been developed to determine this flux and its effects on surface etching of a silica/photoresist combination. The geometry of the source and substrate is very similar to a typical deposition geometry with single or planetary substrate rotation. The model was used to tune an inert ion-etching process that used single or multiple Kaufman sources ${ }^{1}$ to less than $3 \%$ uniformity over a $30-\mathrm{cm}$ aperture after etching $8 \mu \mathrm{m}$ of material. The same model can be used to predict uniformity for ion-assisted deposition (IAD).

In Fig. 1 an ion source is placed in some arbitrary location and orientation. The total ion beam flux seen by a point on a substrate at some radius $\mathrm{r}$ from the center can be approximated by-

$E(r)=\sum_{\phi=0}^{\phi_{\max }} I(\phi, r) R(\phi, r) \Delta \phi$,

where $I(\phi, r)$ is the ion flux intensity determined at point $\phi, r$ and $R(\phi, r)$ is the sputter yield at the same point. $R$ is actually a function of a single variable, the incidence angle of the ion ${ }^{2}$, but both $\phi$ and $r$ are required to determine this angle. In reality, point $P$ will see a range of incidence angles due to the broad nature of the source. Here, a point source is assumed using the top center of the ion-source grid as the origin of ions, and a single incidence angle is used for the approximation. The example in the

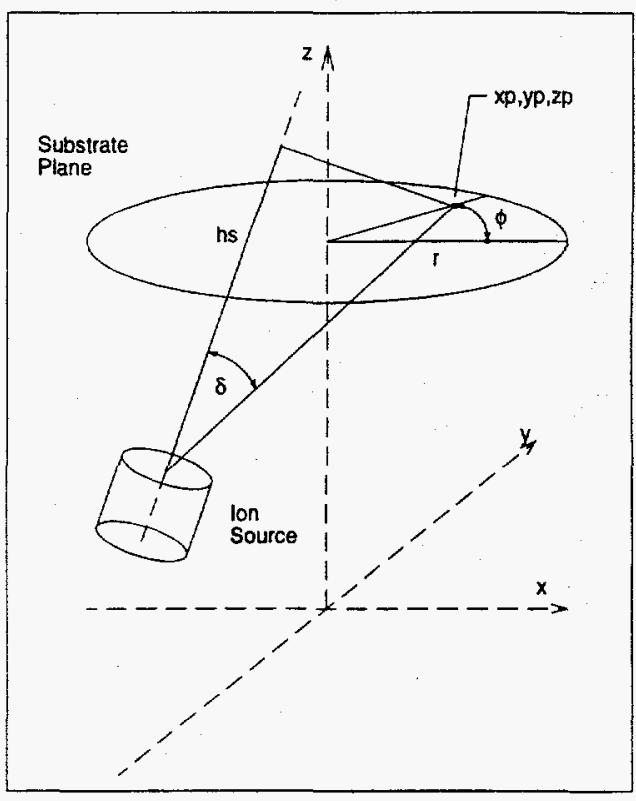

Figure 1. Geometry of the ion source and substrate. diagram shows a simple single rotation of the substrate. A more complex double rotation (planetary) can also be modeled. In this case the point $P$ traces out an epicycloid instead of a circle.

The model described by Eq. (1) can be broken down into three major parts:

(1) Summation and Location Routine: simulates position of a point on a substrate, finds all position and angular parameters, and integrates the calculated flux through some amount of substrate rotation, 


\section{DISCLAIMER}

This report was prepared as an account of work sponsored by an agency of the United States Government. Neither the United States Government nor any agency thereof, nor any of their employees, makes any wartanty, express or implied, or assumes any legal liability or responsibility for the accuracy, completeness, or usefulness of any information, apparatus, product, or process disclosed, or represents that its use would not iniringe privately owned rights. Reference herein to any specific commercial product, process, or service by trade name, trademark, manufacturer, or otherwise does not necessarily constitute or imply its endorsement, recommendation, or favoring by the United States Government or any agency thereof. The views and opinions of authors expressed herein do not necessarily state or reflect those of the United States Government or any agency thereof. 


\section{DISCLAIMER}

Portions of this document may be illegible in electronic image products. Images are produced from the best available original document. 
(2) Expression for $I(\phi, r)$ : a model for the expected ion flux that is determined from measured values of the ion source, and

(3) Expression for $R(\phi, r)$ : a relationship between the incident ion angle and energy and the sputtering yield (or etch rate) of the substrate. This also must be determined experimentally.

The model for $I(\phi, r)$ assumes a rotational symmetry of ion density about the axis normal to the ion-source grids. ${ }^{3}$ This is a reasonable assumption if the discharge chamber in a hot-cathode source is maintained and cleaned regularly. At a given height above the source, the data can then be fit to a 1-D super-gaussian equation

$$
I=a+b e^{\left(-\left|\frac{r-c}{d}\right|\right)^{e}}
$$

where $I=$ ion flux/area for a given $r$

$a=\mathrm{dc}$ offset (usually set to zero)

$b=$ amplitude factor at $r=0$

$c=$ offset for $r$ (set to zero for well-centered beams)

$d=$ width factor

$e=$ shape factor

The factors $b, d$, and $e$ all vary with the height above the source. In practice the beam profile is measured at various heights and each profile is fit to Eq. (2). Then, the values found for $b, d$, and $e$ at various heights are fit to curves using a commercial curve-fitting program. The resulting equations and associated coefficients can then be written into a concise code for modeling. Some results of the measured values and the model are given below in Figs. $2 \mathrm{a}$ and $2 \mathrm{~b}$. The values are for a $16-\mathrm{cm}$ Kaufman source operated with a beam voltage of $300 \mathrm{~V}$ and a total current of $250 \mathrm{~mA}$.

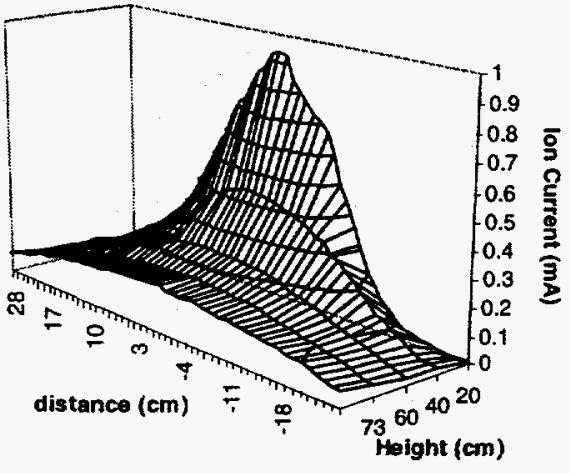

Figure 2(a). Ion flux measurements of a 16-cm ion Figure 2(b). The gaussian model found for the source at different heights above source.

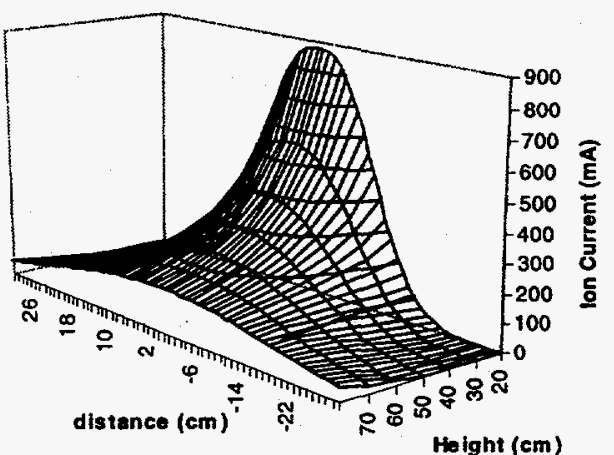

measurements of the $16-\mathrm{cm}$ source at right.

The expression for $R(\phi, r)$, the etch rate as a function of ion incidence angle, was also determined experimentally. Small silica plates with binary photoresist patterns were mounted on miniature rotation drives with the axes set at $20^{\circ}, 30^{\circ}, 40^{\circ}, 50^{\circ}, 60^{\circ}$, and $0^{\circ}$ to 
the beam axis. The center of each substrate was placed on a circle, and the entire assembly was rotated about the circle. Thus, with the ion source on-axis, the ion etch rate for silica and photoresist could be determined simultaneously for six incidence angles by measuring step heights and photoresist thickness. Data was obtained for different operating source parameters and photoresist preparation processes. The normalized data from these experiments was fit to a polynomial of the form $y=l+n \theta^{2}+o \theta^{2.5}+q \theta^{3}$. The two materials did etch at different rates but appear to follow the same curve.

Typical results from the full model are shown in Fig. 3. The array of curves demonstrates the effect on uniformity of moving the source on a radial path away from the substrate. The source axis is at $40^{\circ}$ with respect to the substrate axis, and the source is pointed away from the center. The program also tracks total current for the starting point, which is used to determine the efficiency of a given geometry. Figure 3 shows that a uniformity of better than $7 \%$ could be achieved with careful pointing of the source. In practice, etch uniformity of less than $2.5 \%$ was measured, and in production of $6030-\mathrm{cm}$ optics, uniformity was kept below $6 \%$.

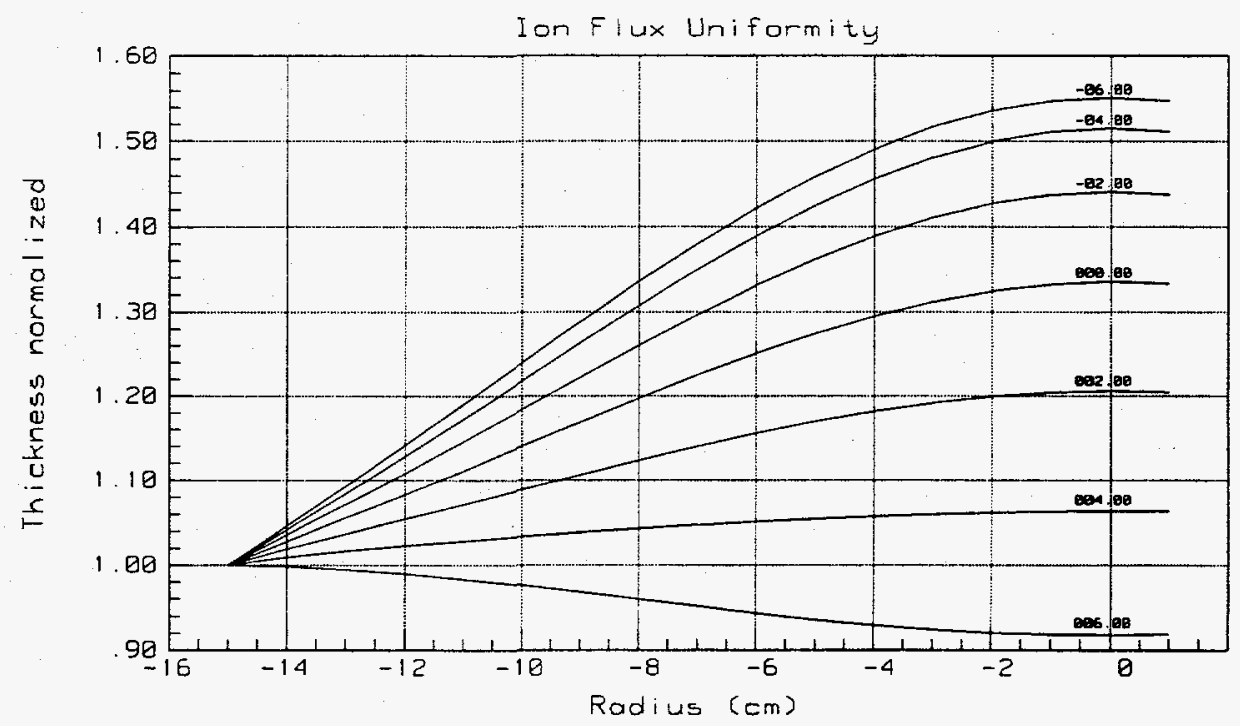

Figure 3. Integrated ion flux as a function of radius across a substrate. Several curves show the effect of moving the ion source away from the substrate center. The source axis is $40^{\circ}$ from vertical and is pointed about $10 \mathrm{~cm}$ away from center.

This work was supported by the U.S. Department of Energy Office of Inertial Confinement Fusion under Cooperative Agreement No. DE-FC03-92SF19460, the University of Rochester, and Lawrence Livermore National Laboratory under subcontract B326760. The support of DOE does not constitute an endorsement by DOE of the views expressed in this article.

${ }^{1}$ Commonwealth Scientific Corporation, Alexandria, VA.

${ }^{2}$ O. Auciello and R. Kelly, Ion Bombardment Modification of Surfaces, (Elsevier, New York, 1984), Chap. 1 and Chap. 5.

${ }^{3}$ M. Jin "Numerical Estimation for 8-cm Ion Source Distribution", Internal Memo, Laboratory for Laser Energetics, University of Rochester, 8 August 1991. 